\title{
Local or systemic metronidazole with scaling and root planing does not increase clinical attachment level
}

Palmer RM, Matthews JP, Wilson RF. Adjunctive systemic and locally delivered metronidazole in the treatment of periodontitis: a controlled clinical trial. Br Dent J 1998; 184:548-552

Objective To test whether treatment of periodontal disease with scaling and root planing plus metronidazole, compared with scaling and root planing alone, increases clinical attachment.

Design A randomised single blind controlled trial.

Intervention Ninety subjects stratified for periodontitis disease severity and smoking status divided into three groups: 1 . Subgingival scaling using ultrasonic scalers and LA; 2. As group 1 plus 7 days of systemic metronidazole (200 $\mathrm{mg}$ tds); 3 . As group 1 plus two applications of $25 \%$ metronidazole gel 1 week apart in all sites with probing depths more than $4 \mathrm{~mm}$.

Outcome measures Mean attachment level gain and the decrease in mean percentages of sites with pocket depth greater than $4.6 \mathrm{~mm}$ six months after therapy.
Results Use of local or systemic metronidazole resulted in no significant additional benefit over scaling and root planing alone. Mean attachment level gain $(\mathrm{mm})$ ranged from 0.47 (for metronidazole gel) to 0.51 for scaling and root planing to 0.67 (for systemic metronidazole), and did not differ significantly from one another.

\begin{tabular}{lllll}
\multicolumn{5}{c}{ Decrease in \% sites with pocket depth $>4.6 \mathrm{~mm}$} \\
& CER & EER & ARR & NNT \\
\hline Scaling + Topical & 10.4 & 11.05 & 0.65 & 153 \\
Scaling + Systemic & 10.4 & 10.71 & 0.31 & 323
\end{tabular}

Conclusions The use of adjunctive metronidazole with scaling and root planing does not increase clinical attachment when compared with scaling and root planing alone.

Address for reprints: RM Palmer, Department of Periodontology and Preventive Dentistry, United Medical and Dental Schools of Guy's and St Thomas' Hospitals, London SE1 9RT UK.

\section{Commentary}

This paper directly tests the clinical and statistical efficacy of adjunctive 'specific' therapy. The study is well-balanced, controlled, blinded, and stratified for disease severity, smoking, and drug delivery mechanism. It evaluates the following variables at 6 months after therapy: plaque, bleeding, pocket depth, attachment level, and indirectly assesses the infection. Their findings indicate that, for patients with either moderate or severe periodontal disease, adjunctive metronidazole (provided either systemically or locally) does not enhance the benefit of scaling and root planing.

The study has two significant limitations. First, it does not control for compliance. This is important because the drug has significant effects on the gastrointestinal tract, alters taste, and prevents compliant patients from imbibing alcohol. Second, the microbiological assessment is by darkfield microscopy. This is an inaccurate measure of pathogenicity, and might have been supplemented by a microbial test (e.g. BANA test) specific for the small set of bacterial pathogens. One could argue that both of the foregoing reservations are controlled for by the use of metronidazole gel. A search of Medline from 1996 to the present, however, identified only three randomized controlled trials comparing scaling with scaling plus metronidazole gel, and all three indicated that the gel provided little clinical improvement over scaling alone (e.g. Kinane and $\operatorname{Radvar}^{1}$ ). The gel may thus be a poor control for systemic metronidazole.

The results also conflict with a series of clinical trials by Loesche ${ }^{2}$ and his colleagues who found that adjunctive use of metronidazole results in a $<80 \%$ reduction in the need for surgery. On the other hand, this study supports the metaanalysis findings ${ }^{3}$ that adjunctive metronidazole use offers little clinical benefit.
Given these sometimes conflicting results, the preponderance of best evidence indicates that metronidazole, when used as an adjunctive to scaling and root planing, offers little clinical benefit in treating periodontal disease.

1. Kinane DF, Radvar M. A six-month comparison of three periodontal local antimicrobial therapies in persistent periodontal pockets. J Periodontol 1999. 70:1-7.

2. Loesche WJ, Giordano J, Soehren S, Hutchinson R, Rau CF, Walsh L, Schork $M A$. Nonsurgical treatment of patients with periodontal disease. Oral Surg Oral Med Oral Path 1996; 81:533-543.

3. Elter JR, Lawrence HP, Offenbacher S, Beck JD. Meta-analysis of the effect of systemic metronidazole as a adjunct to scaling and root planing for adult periodontitis. J Periodont Res 1997; 32:487-496.

\section{Richard Niederman}

Harvard School of Dental Medicine, Boston, USA 